\title{
Strongyloides as a Cause of Fever of Unknown Origin
}

Iliana Neumann, MD, Rhianna Ritter, MD, and Anne Mounsey, MD

Strongyloides is endemic in parts of the United States. Most often it is asymptomatic but it has a wide range of clinical presentations. Because of the unusual capacity of strongyloides for autoinfection, it can cause hyperinfection, when it effects the pulmonary and gastrointestinal systems, or disseminated infection, when other organs are involved. Both hyperinfection and disseminated strongyloides usually occur in immunosuppressed patients. We report a case of hyperinfection with strongyloides in a man presenting with fever of unknown origin who was not immunosuppressed. (J Am Board Fam Med 2012;25: 390-393.)

Keywords: Case Report, Fever of Unknown Origin, Infectious Diseases, Strongyloides

A 36-year-old Hispanic man presented with 4 weeks of fever up to $38.8^{\circ} \mathrm{C}$, a nonproductive cough, generalized abdominal pain, and a $12-\mathrm{lb}$ weight loss. He had no history of tick exposure, contact with sickness, or recent travel. He had been treated previously with antiviral medication for influenza and with ciprofloxacin for presumptive prostatitis with no improvement in his symptoms. He had no significant medical history and was not taking any chronic medications. He worked as a brick layer and lived with his wife and children in rural North Carolina; he denied alcohol use. Review of systems was negative for diarrhea, vomiting, rash, night sweats, and dysuria. It was positive for headache, malaise, myalgias, arthralgias, and decreased appetite.

Physical examination revealed a well-nourished, well-developed man who appeared flushed but was otherwise in no acute distress. He had a temperature of $38.6^{\circ} \mathrm{C}$, pulse of 95 beats per minute, respiratory rate of 22 breaths per minute, and blood pressure of 146/83 $\mathrm{mm} \mathrm{Hg}$. Physical examination

This article was externally peer reviewed.

Submitted 28 March 2011; revised 11 July 2011; accepted 9 August 2011.

From the Department of Family Medicine, University of North Carolina, Chapel Hill.

Funding: none.

Conflict of interest: none declared.

Corresponding author: Anne Mounsey, MD, 590 Manning Drive, Chapel Hill, NC 27599 (E-mail: anne_mounsey@ med.unc.edu). was otherwise normal. Laboratory examination revealed a white blood cell count of $11.2 \times 10^{9} / \mathrm{L}$ (normal, 4.5 to $11 \times 10^{9} / \mathrm{L}$ ); neutrophils, $8.1 \times$ $10^{9} / \mathrm{L}$ (normal, 2.0 to $7.5 \times 10^{9} / \mathrm{L}$ ); lymphocytes, $2.2 \times 10^{9} / \mathrm{L}$ (normal, 1.5 to $5.0 \times 10^{9} / \mathrm{L}$ ); monocytes, $0.5 \times 10^{9} / \mathrm{L}$ (normal, 0.2 to $0.8 \times 10^{9} / \mathrm{L}$ ); eosinophils, $0.2 \times 10^{9} / \mathrm{L}$ (normal, $<0.4 \times 10^{9} / \mathrm{L}$ ); hematocrit, $34.7 \%$ (normal, $36 \%$ to $46 \%$ ); alanine transaminase, $71 \mathrm{U} / \mathrm{L}$ (normal, 15-38 U/L); aspartate transaminase, $87 \mathrm{U} / \mathrm{L}$ (normal, 14-38 U/L); alkaline phosphatase, $204 \mathrm{U} / \mathrm{L}$ (normal, 38-126 $\mathrm{U} / \mathrm{L}$ ); and a sedimentation rate of 24 . Chest roentogram revealed a subtle left basilar opacity. Computed tomography of the chest showed bibasilar opacities.

The patient was admitted to the hospital and had an extensive work up for fever of unknown origin including rheumatologic, infectious, and malignant etiologies. Serology for human immunodeficiency virus and acute hepatitis $\mathrm{A}, \mathrm{B}$, and $\mathrm{C}$ infection was negative. Blood, urine, and cerebrospinal fluid cultures were all negative. Stool microscopy revealed the larvae of Strongyloides stercoralis.

Strongyloides stercoralis is discussed here because it needs to be included in the differential diagnosis of fever of unknown origin. In many diagnostic lists of fever of unknown origin, helminth infections are not mentioned. ${ }^{1}$ Strongyloides, especially in its hyperinfective or disseminated forms, causes fever and significant morbidity and mortality. Diagnosis requires a high index of 
Figure 1. Computed tomography scan of the patient's chest, showing bilateral basilar opacities greater on the right than the left.

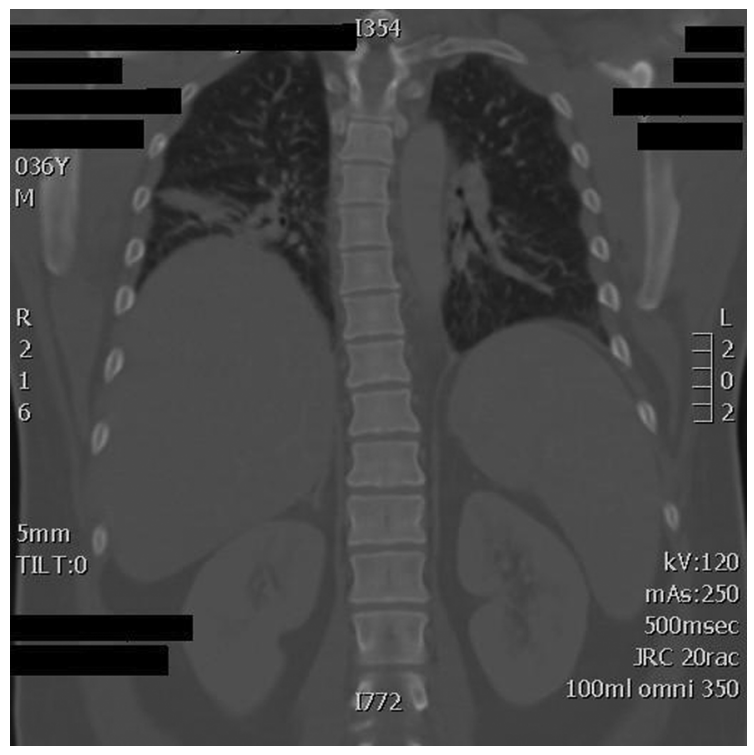

suspicion because presenting symptoms are nonspecific. The most common presenting symptom in severe disease is fever, present in $100 \%$ of cases in one study of disseminated disease. ${ }^{2}$

\section{Literature Review}

Our case study is unusual in that our patient was an otherwise healthy man who presented with hyperinfection strongyloides defined by fever, respiratory symptoms, and pulmonary infiltrates on imaging studies with strongyloides in his stool. A PubMed literature review was performed and last updated March 16, 2011. One search was performed with the Medical Subject Headings strongyloides and immunocompetent. Thirty-seven articles were found, of which 2 were reports of strongyloides hyperinfection in immunocompetent patients. $^{3,4}$ The references of these 2 reports were reviewed for further publications but none were found.

\section{Discussion}

Endemic areas of strongyloides stercoralis include the southeastern United States, Puerto Rico, Central America, the Pacific Basin, and Central Africa. In rural areas of the southeastern United States, especially the Appalachia region, epidemiologic studies report prevalences from $2.5 \%$ to $4 \% .^{5}$ Prevalences in Latin America and Africa are as high as
$50 \% .^{6}$ Improved sanitation and a population shift to cities has decreased helminth infections in the United States, but they continue to be a public health burden in less developed parts of the world. Disease from strongyloides has a broad spectrum of clinical course. It can be asymptomatic, acute, or chronic with pulmonary or gastrointestinal symptoms. Rarely, strongyloides can be disseminated or cause hyperinfection, particularly in the immunosupressed.

Strongyloidiasis is caused by an intestinal parasitic nematode. The species, Strongyloides stercoralis, is the most common and clinically important. Its life cycle is more complex than other nematodes because it alternates between free living and parasitic cycles. It also has the potential for autoinfection and multiplication within the host.

In its parasitic cycle, filariform larvae infect the host by contact with human skin, usually the feet through contaminated soil. These larvae invade the cutaneous capillaries and disseminate hematogenously to the pulmonary capillaries, where they penetrate into the alveolar space. The larvae then travel up the bronchial tree to the pharynx, where they are swallowed and reach the small intestine. In the small intestine they become adult female worms that live in the epithelium and produce eggs. The eggs develop into rhabditiform larvae, which are released into the stool.

Rhabditiform larvae are part of the free-living cycle of the nematode and are responsible for the organism's distinctive ability for autoinfection. These larvae can begin a free-living cycle in the soil or they can penetrate the intestinal mucosa or the skin of the perianal area to cause autoinfection and eventually disseminate widely in the body. This ability to auto infect allows strongyloides to persist and replicate within a host for decades. In patients with normal immune systems, this replication is kept under control, but in immunosuppressed patients, autoinfection can lead to hyperinfection and disseminated strongyloides. ${ }^{7}$

\section{Clinical Manifestations}

Most patients infected with strongyloides are completely asymptomatic. ${ }^{8}$ Some have mild gastrointestinal, cutaneous, or pulmonary symptoms with or without fever. These symptoms may be acute or they may wax and wane chronically before spontaneous resolution. Gastrointestinal presentations include diarrhea and abdominal discomfort, nausea, 
anorexia, weight loss caused by gastritis, or enteritis with ulceration. Patients also can have occult gastrointestinal blood loss or malabsorption of fat and vitamin $\mathrm{B}_{12}{ }^{5}$

Pulmonary symptoms include cough and dyspnea, sometimes associated with wheezing, caused by larval migration through the lungs. Strongyloides is also a cause of eosinophilic pneumonia characterized by pulmonary infiltrates and blood eosinophilia. ${ }^{9}$ Pulmonary manifestations are more common in those with chronic lung disease who are taking oral steroids. ${ }^{7}$

Cutaneous symptoms include pruritic papulovesicular lesions at the site of initial skin penetration, usually the feet. The pathognomonic rash of strongyloides infection is larva currens (racing larvae). It is a serpiginous urticarial rash that creeps up the body 5 to 15 centimeters per hour. It likely represents an allergic response to migrating larvae. In chronic disease with cycles of autoinfection the rash can recur over weeks, months, or even years. ${ }^{10}$

The 2 most severe forms of strongyloidiasis are hyperinfection and disseminated syndromes. These occur most often in patients with impaired cellmediated immunity. Hyperinfection represents excessive worm burden (defined as an amplification of autoinfective life cycle) without the spread of larva outside its usual migration pattern in the gastrointestinal tract and lungs. There is no quantitative definition, but it is characterized by an increase in gastrointestinal or pulmonary symptoms with an increased larval load in the stool or sputum. Disseminated strongyloidiasis involves spreading to other sites such as the heart, urinary tract, central nervous system, and endocrine organs. In disseminated strongyloides, massive larval migration causing vessel injury can lead to a petechial purpuric skin eruption. Mortality with hyperinfection can be up to $87 \%$ in the immunosuppressed. ${ }^{11}$

Risks for developing hyperinfection and disseminated strongyloidiasis include immunosuppressive therapy (most commonly steroids), malignancy, malabsorption states, end-stage renal disease, diabetes mellitus, advanced age, or HIV infection. ${ }^{12}$

\section{Diagnosis}

Diagnosis is made by observation of the larvae in sputum or stool specimens. In chronic infection, at least 3 stool samples on consecutive days must be taken because larval output is low and intermittent. Sensitivity with a single stool sample is only $30 \%$ but increases to $70 \%$ to $85 \%$ with 3 consecutive stool samples using the agar plate method of larval detection. ${ }^{13}$ The best method for detecting exposure is the enzyme-linked immunosorbent assay for immunoglobulin G antibodies against $S$. stercoralis with sensitivities between $74 \%$ and $98 \%$ and a specificity of $100 \%{ }^{7}$ However, if positive, this cannot distinguish between past infection and current infection. Eosinophilia is present in $50 \%$ to $80 \%$ of patients with mild infection. In contrast, a low eosinophil count occurs in patients with hyperinfection and disseminated disease. ${ }^{5}$ Chest radiograph can reveal diffuse alveolar or diffuse interstitial infiltrates, segmental alveolar infiltrates, or pleural effusions. ${ }^{14}$ Our patient had a low eosinophil count and mild impairment of liver function tests, transaminases, and alkaline phosphatase, which have been reported in up to $52 \%$ of patients with strongyloidiasis. ${ }^{15}$

\section{Conclusion}

Our patient was unusual in that he was otherwise healthy and not immunosuppressed but presented with fever and pulmonary and gastrointestinal symptoms of strongyloidiasis hyperinfection syndrome. He had risk factors for strongyloides: he lived in the southeastern United States, was an immigrant from Mexico, and worked in brick laying, which may have caused him to have greater exposure to soil.

The patient was treated with albenza $400 \mathrm{mg}$ twice per day for 3 days and ivermectin $3 \mathrm{mg}$ per day for 5 days. There is no general agreement of the best treatment regime for hyperinfection and disseminated strongyloides because of the lack of randomized, controlled studies, but expert opinion recommends ivermectin or a combination of ivermectin and albendazole for 5 to 7 days. Immunosupressed patients may need longer treatment regimes before symptom improvement. $^{16,17}$ Thiobendazole also is effective but albendazole is preferred because of better tolerability. Drug monitoring is not usually done because effective therapeutic levels in humans are unknown. In sick patients, oral absorption may be impaired; in such patients with a poor clinical response to treatment, subcutaneous or rectal ivermectin can be used. Treatment efficacy can be monitored by follow-up stool examinations, usually at 2 weeks and then confirmed at 3 months, because of the low sensitivity of a single stool sample. ${ }^{2,13,16}$ Treatment 
success also can be monitored by decreased eosinophilia and a decreased level of immunoglobulin G strongyloides antibodies. ${ }^{18}$ One week after the initiation of treatment our patient had complete resolution of his fever, abdominal pain, cough, and malaise. Follow-up stool examination at 3 weeks was negative for strongyloides. At 3 months both stool examination and chest radiograph were nor$\mathrm{mal}$, and he remains symptom free 2 years after treatment.

\section{Summary}

Strongyloides infection needs to be considered in the differential diagnosis of fever of unknown origin in people living in or immigrants from endemic areas. Stool examination for parasites should be considered in such patients with a fever and generalized respiratory and gastrointestinal symptoms, even in the absence of diarrhea.

\section{References}

1. Up to Date. Available at: http://www.uptodate.com/ contents/image? imageKey $=\mathrm{ID} / 62509 \&$ topicKey $=$ ID $\% 2$ F2737\&source $=$ outline_link\&utdPopup $=$ true Accessed 8 June 2011.

2. Lam, CS, Tong MK, Cahn KM, Siu YP. Disseminated strongyloides: a retrospective study of clinical course and outcome. Eur J Clin Microbiol Infect Dis 2006;25:14-8.

3. Marathe A, Date V. Strongyloides stercoralis infection in an immunocompetent patient with extreme eosoinophilia. J Parasitol 2008;94:759-60.

4. Sridhara S, Simon N, Raghuraman U, Crowson N, Aggarwal V. Strongyloides stercoralis pancolitis in an immunocompetent patient. Gastrointest Endosc 2008;68:196-9.

5. Concha R, Harrington WJ, Rogers AI. Intestinal strongylodiasis: recognition, management, and determinants of outcome. J Clin Gastroenterol 2005; 39:203-11.
6. Genta R. Global prevalence of strongyloidiasis: critical review with epidemiologic insights into the prevention of disseminated disease. Rev Infect Dis 1989; 11:755-67.

7. Vadlamudi RS, Chi DS, Krishnaswamy G. Intestinal strongyloidiasis and hyperinfection syndrome. Clin Mol Allergy 2006;4:8.

8. Keiser PB, Nutman TB. Strongyloidiasis stercoralis in the immunocompromised population. Clin Microbiol Rev. 004;17:208-17.

9. Gerke AK, Hunninghake GW. Hypersensitivity pneumonitis and pulmonary infiltrates with eosinophilia. Harrisons online edition. Available at: http://accessmedicine.com/popup.aspx?aID = 2869483\&print=yes_chapter. Accessed 8 June 2011.

10. Von Kuster LC, Genta RM. Cutaneuos manifestations of strongyloidiasis. Arch Dermatol 1988;124: 1826-30.

11. Siddiqui AA, Berk SL. Diagnosis of strongyloides stercoralis infection. Clin Infect Dis 2001;33: 1040-7.

12. Nuccio M. Strongyloides in patients with hematologic malignancies. Clin Infect Dis 1995;21:675-7.

13. Sato Y, Kobayashi J, Toma H, Shiroma Y. Efficacy of stool examination for detection of strongyloides infection. Am J Trop Med Hyg 1995;53:248-50.

14. Chu E. Pulmonary hyperinfection syndrome with strongyloides stercoralis. Chest 1990;97:1475-7.

15. Tsia HC, Lee SS, Liu YC, et al. Clinical manifestations of strongyloidisis in southern Taiwan. J Microbiol Immunol Infect 2002;35:29-36.

16. Kabirdas, D, Alfonso B, Avella H, Kanwar A, Berho M, Oliviera E. An elderly woman with asthma, eosinophilia, and septic shock. Cleve Clin J Med 2007; 74(12):877-85.

17. Segarra-Newnham M. Manifestations, diagnosis, and treatment of strongyloides stercoralis infection. Ann Pharmacother 2007;41:1992-2001.

18. Loutfy MR, Wilson M, Keystone J, Kain K. Serology and eosinophil count in the diagnosis and management of strongyloidiasis in a non endemic area. Am J Trop Med Hyg 2002;66(6):749-52. 